\title{
Comparison of Cerebral Artery Angle in Adults and Fetuses
}

\author{
Comparación del Ángulo de Arteria Cerebral en Adultos y Fetos \\ Feng Fan*; Tao Xiang***; Jiaywei Tsauo**; Xiaodong Xie ${ }^{* * * * *} \& \mathrm{Xiao} \mathrm{Li}^{* * *}$
}

FAN, F.; XIANG, T.; TSAUO, T.; XIE, X. \& LI, X. Comparison of cerebral artery angle in adults and fetuses. Int. J. Morphol., 33(3):942-947, 2015.

SUMMARY: In this study we generated casting specimens of human cerebral blood vessels for twenty-five fetuses and thirtysix adults. The degrees of the angles were measured and comprised for those blood vessels frequently involved in the interventional catheterization.

KEY WORDS: Cerebral artery angle; Interventional catheterization; Specimens.

\section{INTRODUCTION}

The rapid evolution and refinement of newer technologies have not only made it possible to broaden and diversify our approach in better diagnosing and treating an increasing number of different diseases but also revolutionized the role played by interventional radiology as an indispensable component in diagnosing and treating cerebrovascular diseases (Mordasini et al., 2005; Turk et al., 2007; Wang et al., 2008). While planning and performing any management procedure involving interventional the most basic, nevertheless the most crucial venture is introducing the diagnostic and/or the therapeutic catheter. This is easily verified by the fact that if we retrospectively analyze the possible reasons for any failure and/or complications that might have occurred during the catheterization, they can be reduced to only one: the interventionist not being sufficiently acquainted with the formation of different kinds of angles in between various blood vessels and their respective branches, which by itself, is a very complicated and diversified meshwork. The inability to precisely predict the range of an angle that one or more arteries make with different other arteries make the task of producing a corresponding appropriate chamber or mold for the head end of the guide wire indeed a very demanding and exacting one. However, as of yet, no detailed reporting dealing with the catheterization-related angle formation of cerebral blood vessels has been available. Therefore, in this study we generated casting specimens of human cerebral blood vessels for twenty-five fetuses and thirty-six adults. The degrees of the angles were measured for those blood vessels frequently involved in the interventional catheterization.

\section{MATERIAL AND METHOD}

Generation of casting specimens for the cerebral blood vessel in the fetuses. All the usage of samples related to the fetuses was approved by the Ethics Committee of NO. 2 West China Hospital, Sichuan University. The fetus samples were provided by NO.2 West China Hospital, Sichuan University.

A total of 25 fetus specimens (fetus age: 5-6 months) were collected by separation from the seventh cervical vertebra. An interventional catheter (3-6F, Teramo Co., Fijinomiya, Japan) was inserted into one of the common carotid arteries followed by several flushes with clean pressurized water. Red fluid filling agent (acetone: acrylonitrile-butadiene-styrene=1:1, Qimei, Guangdong, China) was then perfused until the filling agent overflowed out of the other common carotid artery as well as both sides of vertebral artery. The pressure within the artery maintained for approximately 5-10 min. If, by any chance, the filling agent only overflowed out of the other common carotid artery

\footnotetext{
* Department of Interventional Neuroradiology, The First Affiliated Hospital of Zhengzhou University, Zhengzhou, China.

** Institute of Interventional Neuroradiology, West China Hospital, Sichuan University, Chengdu, China.

*** Teaching Lab. of Stomatology, West China Hospital of Stomatology, Sichuan University, Chengdu, China.

***** Department of Neurosurgery, West China Hospital, Sichuan University, Chengdu, China.
} 
or only overflowed out of either sides or only one side of the vertebral artery, then the filling agent was re-perfused into the other common carotid artery until the filling agent overflowed out of both the vertebral arteries. The volume of the filling agent was approximately 5-20 ml. After, half an hour to one hour of perfusion, the specimens were corroded with the help of $31 \%$ of industrious hydrochloric acid (Hengyang, Hunan, China).

They were further preserved for about 1-2 weeks after which they were flushed and trimmed for observation.

Generation of casting specimens for the cerebral blood vessels in the adults. The adult specimens $(n=36)$ were provided by the Department of Anatomy, West China Forensic and Basic Medical College, Sichuan University. Use of these specimens was approved by Ethics Committee of West China Hospital, Sichuan University.

A total of 36 adult specimens (including head and neck) that were separated from the third or fourth cervical vertebra were regularly fixed. The common carotid artery or internal carotid artery were intubated and flushed with water for several times in order to maximally avoid the influence on permeation due to large amount of blood clots. The filling agent was perfused through a catheter. If the filling agent was leaking out of the blood vessels during the process of perfusion, the leaking point would be either ligated or clamped by using haemostatic forceps. The volume of the filling agent was approximately $15-25 \mathrm{ml}$. Once the perfusion was completed, the specimens were completely immersed upside down in a glass container containing $31 \%$ of industrious hydrochloric acid. After 5-7 days, the specimens were washed. Hair, corroded tissues in the skin, neck and face were carefully removed. Fresh acid was again used to corrode the specimens for approximately 2 weeks before it was taken out. After washing with flowing water for 12-24 hours, the soft tissues and decalcified bones in the bottom of the skull were removed. The internal carotid artery and vertebral artery were maintained for observation.

Observation for the direction of the artery bifurcation and measurement the angles between major arteries and their branches. The anatomical position was used as the criterion for measuring the angles made by the branching arteries. The following approaches were used to measure the angles. The intersection of the two blood vessels was designated as vertex $(\mathrm{C})$ of the angle. Two arbitrary points, (A) and (B), were, respectively, selected from both blood vessels that were on the same parallel surfaces. The distance between A and C, B and C, A and B was precisely measured. The angle was calculated applying cosine theorem.
Data analysis. A comparison of the angles between the adult and the fetus group reveled that there was a statistical significant difference of $\mathrm{P}<0.05$ in the student $t$ test. Software SPSS10.0 was used for all the statistical analysis.

\section{RESULTS}

Angle formation between internal carotid artery (ICA) and ophthalmic artery (OA) (C6-OA). OA is the first branch of the encephalic internal carotid artery. In the current study, the number of the OA that originated from the anterior wall of the ICA and the upper wall of the ICA accounted for $47.7 \%(31 / 65)$ and $52.3 \%(34 / 65)$ respectively. In $56.4 \%$ of the OA, a short artery was branched in the inner-anterior or direct-anterior direction and then turned back into the antero-lateral and inner side of the orbit. This short artery was approximately $05-2.0 \mathrm{~mm}$ in length and formed an acute, right or obtuse with the distant OA. Approximately $43.1 \%$ of the OA did not have a branched short artery and entered the orbit directly through the antero-lateral direction. The angle between the short artery and the $\mathrm{C} 6$ or between starting point of OA and C6 was measured. The vertex of this angle was the intersection of OA and C6. One arm of the angle was formed by the OA while the other arm was formed by the ICA below the vertex (Fig. 2: 5). This angle usually pointed towards the postero-lateral direction and was obtuse (Table I).

Angle formation between internal carotid artery and anterior choroidal artery (C7-AChA). Anterior choroidal artery (AChA) originated from the $\mathrm{C} 7$ portion of the ICA and a total of 110 AchA were identified from both groups. Among these 110 AchA, 84.5\% (93/110) and 15.4\% (17/ 110) branched out respectively from the posterior wall and the lateral walls of the ICA. The angle between C7 and AchA was normally pointed toward the antero-inferior direction. The vertex of the angle was the intersection between AchA and C7. While one arm of the angle was formed by the AchA, the other arm was formed by the ICA between the vertex and PCoA (Fig. 2, 3). The degrees made by the C7-AChA are shown in Table I. It was found that, this angle would normally be an obtuse one in the adult group in contrast to an acute one in the fetus group $(\mathrm{P}<0.05)$.

Angle formation between the A1 portion of the anterior cerebral artery and the anterior communicating artery (ACoA) (A1-ACoA). ACoA is the blood vessel that connects the right and left sides of the ICA. ACoA can be a single or multiple vessels that are horizontally or obliquely crossing between ICAs, thus contributing to the formation of the anterior portion of the Cerebral arterial circle. Owing to the 
FAN, F.; XIANG, T.; TSAUO, T.; XIE, X. \& LI, X. Comparison of cerebral artery angle in adults and fetuses. Int. J. Morphol., 33(3):942-947, 2015.

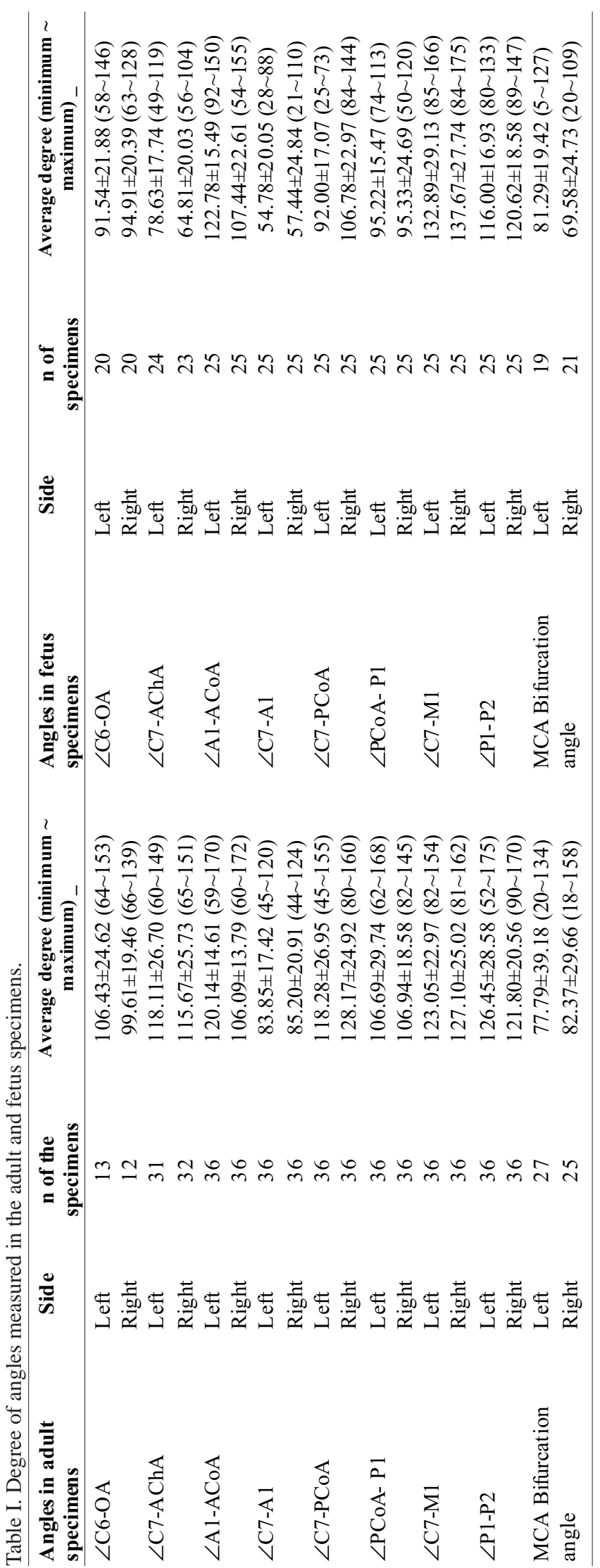

relative complexity of the arterial meshwork, the blood vessels had a higher number of possible variations of presentation and the two arms of the angle would not always be on the same plane as they were at the point of intersection (vertex) of the A1 portion and the ACoA; thus making it possible to only measure the angle nearby the vertex. If there were multiple ACoA, we measured the most proximal ACoA (Fig. 1, 1). It was also revealed that the position of the A1 was relatively stable in comparison to the ACoA. Depending on the variation of the two contributing arms, this angle could be pointed towards the antero-interior, antero-lateral or in any other direction. Normally, A1-ACoA was an obtuse angle.

Angle formation between the internal carotid and the $A 1$ portion of the anterior cerebral arteries (C7A1). ICA was branched into ACA and MCA below the prae cerebrum. ACA was a relatively small branch. C7 of ICA formed an angle with A1 portion of ACA. The vertex of the angle was the intersection between $\mathrm{C} 7$ and A1. The two contributing arms were the A1 portion and the ICA between the vertex and AChA (Fig. 1,2). The angle was pointed towards the posterior or antero-lateral direction. Normally, C7-A1 was an acute angle. C7A1 in the adults were significantly larger than that in the fetuses $(\mathrm{P}<0.05)$ (Table I).

Angle formation between the $\mathrm{C} 7$ portion of internal carotid artery and the posterior communicating artery (PCoA) (C7-PCoA). C7 portion of the ICA formed an angle with the PCoA. The vertex of the angle was the intersection between the $\mathrm{C} 7$ and the PCoA. The contributing arms were the PcoA and the ICA between OA to the vertex (Fig. 2,4). This angle normally pointed towards the antero-superior or antero-inferior direction. Usually, C7 formed an obtuse angle with PCoA. The C7-PCoA was significantly larger in the adults than in the fetuses. $(\mathrm{P}<0.05)$ (Table I).

Angle formation between the posterior communicating (PCA) and the P1 portion of posterior cerebral arteries (PCoA-P1). It was observed that the PCoA merged with the proximal portion of the PCA, forming the posterior part of the Cerebral arterial circle. PCoA formed an angle with P1. The vertex of the angle was the intersection between PCoA and P1, thus making the P1 and the PCoA the contributing arms (Fig. 3, 8). This angle was pointed towards the postero-lateral direction. PCoA-P1 was normally an obtuse angle (Table I).

Angle formation between the internal carotid artery and the M1 portion of the middle cerebral artery 


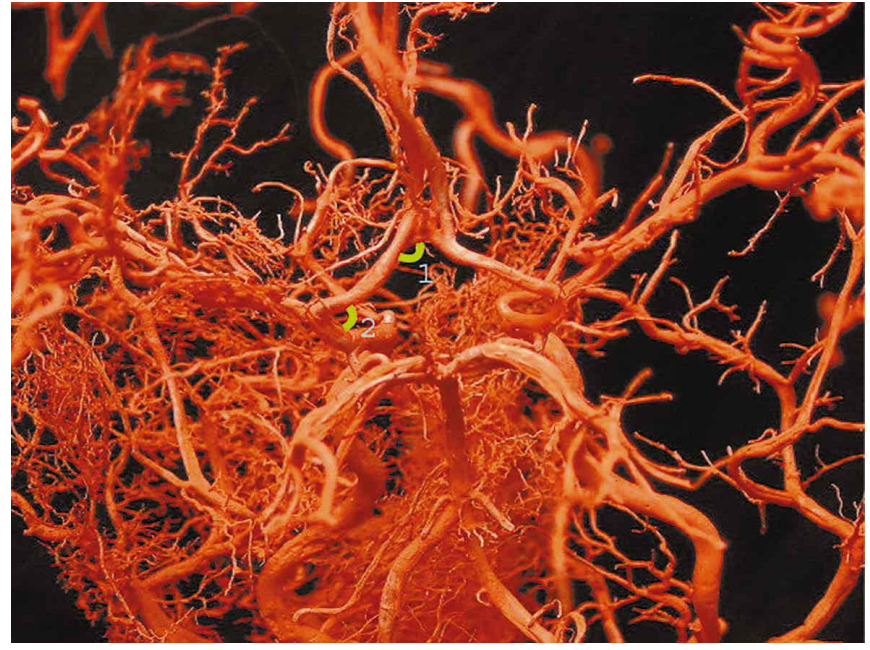

Fig. 1. 1 and 2 represent A1-ACoA and C7- A1, respectively.

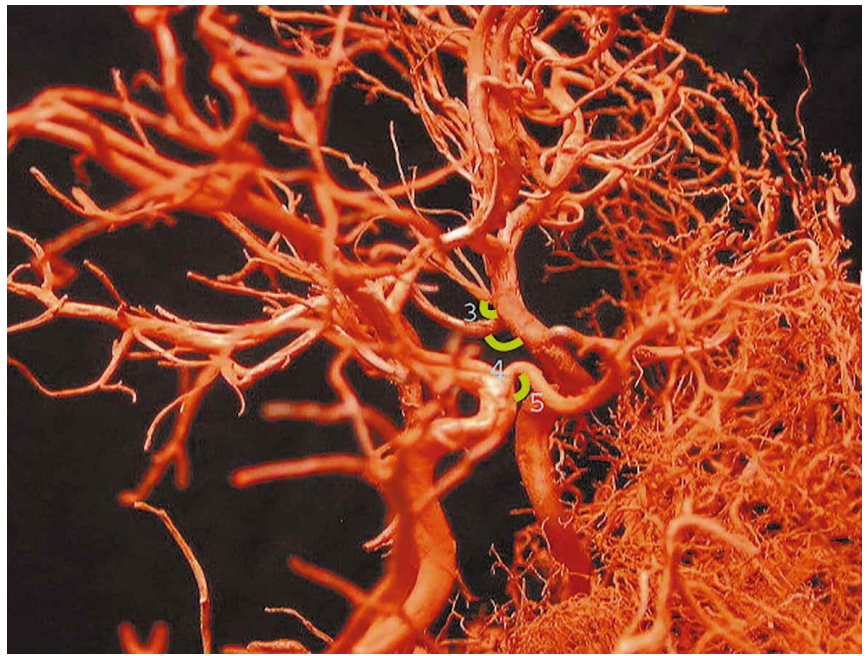

Fig. 2. 3, 4 and 5 represent C7- AChA, C7- PCoA and C6-OA, respectively.

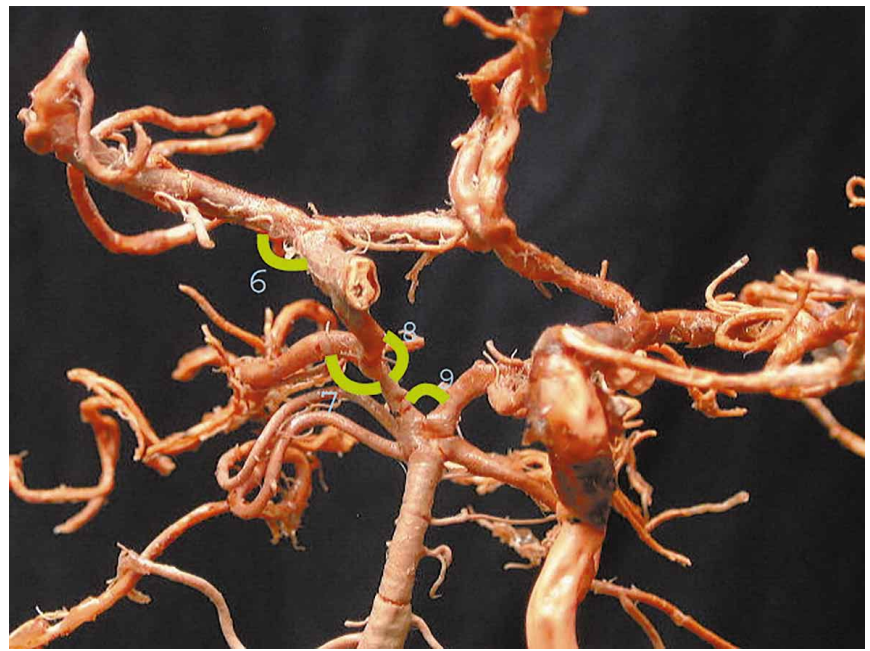

Fig. 3. 6, 7, 8 and 9 represent C7- M1, P1- P2, PCoA-P1 and BA, respectively.
(MCA) (C7-M1). C7 of the ICA formed an angle with theM1 portion of the MCA. The vertex of the angle was the intersection between $\mathrm{C} 7$ and M1. An arm of the angle was made by the M1 while another was formed by the ICA between vertex and AchA (Fig. 3, 6 ). This angle pointed towards the postero-inferior or the postero-anterior direction. MCA was a relatively larger branch of two ICA branches, which equaled to the continuous portion of ICA. Therefore, C7-M1 was usually an obtuse angle (Table I).

Angle formation between the $\mathrm{P} 1$ and $\mathrm{P} 2$ portions the of posterior cerebral artery (P1-P2). The vertex of P1-P2 was the intersection between P1 and P2 (Fig. $3,7)$. This angle pointed towards the antero-lateral direction and was usually obtuse (Table I). The PCAs on both sides were the final branches of basilar artery (BA). Once branched out from the BA, the PCAs stretched out toward the postero-lateral direction and merged with the PCoA. The P1 portion was also recognized as the mesencephalon portion or the anterior portion of the communicating artery. P1 portion represented the most proximal portion of the PCA and was seen bending around the mesencephalon towards posterior-lateral direction. P2 portion was also named an ambient cisternal portion. P2 was the last portion between the merging site and the mesencephalonian posterior portion.

The angle of bifurcation formed by the M1 portion of middle cerebral artery (M1). It was seen that, as the middle cerebral artery (MCA) entered the area of the insula, it divided into two to three branches, only two branches being the most frequent study, thus implying that only the angle of bifurcation at the M1 portion of the MCA, had to be measured; and this angle usually pointed towards the intero-interior or the postero-interior direction (Fig. 4, 10, Table I).

The angle formed by the basilar artery bifurcation (BA). Basilar artery (BA), which lies almost at the midline, is formed by the combination of the two vertebral arteries near the junction of the pons and the medulla oblongata and usually ends by giving rise to two posterior cerebral arteries; thus resulting in an angle at the point of emergence of these two posterior cerebral arteries, the arms of which were namely the left and the right sided P1 portions, and which was usually found to be obtuse pointing towards the inferior direction (Fig. 3, 9). BA measured in 36 adult specimens was $122.60 \pm 37.08(60 \sim 175)$, in contrast to $133.33 \pm 35.19$, in the 23 fetuses (66 177). 


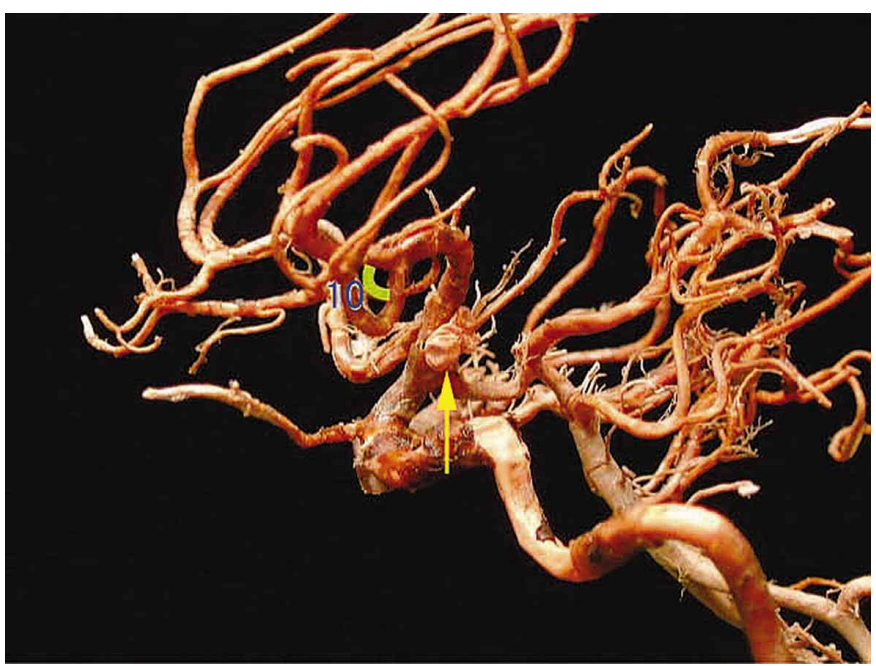

Fig. 4. 10 is double-stem MCA bifurcation angle. The arrow indicates the aneurysm at the starting sites of anterior choroidal artery.

\section{DISCUSSION}

Vascular branches and angle formation. Due to the extreme complexity of the cerebral vascular branches and angle formation, we only measured the degrees of the angles formed by the blood vessels that are frequently catheterized in clinical interventional diagnostic and therapeutic scenarios. As we have seen above, the obtuse angles to be found in both the adult and fetal specimens were C6-OA, ACoA-A1, C7-PCoA, PCoA-P1, C7-M1, P1-P2, while the C7-AChA and the BA were normally obtuse only among the adults. Likewise, we observed that the acute angles were M1, C7-A1, and C7$\mathrm{AChA}$; the first two being acute in both the categories while the latter being acute only among the fetuses. The average magnitudes of the C7-A1, C7-AChA, and C7-PCoA were significantly greater in the adults than in the fetuses. In addition, the adults and the fetuses had respectively an obtuse and an acute $\mathrm{C} 7-\mathrm{AChA}$. These results are suggestive of relatively greater alternations in the $\mathrm{C} 7-\mathrm{A} 1, \mathrm{C} 7-\mathrm{AChA}$ and C7-PCoA during the course of human development from fetus to adulthood. During the fetal period, C7 portion of the internal carotid artery is originates from the extension of the aorta on the dorsal side while the PCoA originates from the degeneration of internal carotid artery. In the mean time, the developing and bending of the cerebral hemisphere may also have a certain effect on the vascular angle formation. Significant difference in the magnitude of the C7-AChA in the two groups can probably be resultant adaptation due to the development of the choroid plexus and the telencephalon. It could also have been precipitated by the difference in the ratio of the neurocranial and the cranial viscera in between the two groups. We see an increase in the magnitude of the angle formed by AChA and ICA along with the enlargement of the cerebral hemisphere (Moore, 1977). From the perspective of species embryology, AChA is originally the communicating vessel between ICA and PCA. Along with the unraveling of evolution, we can notice that the choroid plexus developed into a more prominent structure, as did the formation of the AChA, resultant to the gradual degeneration of the connection between the communicating vessel and the PCA. It is not too uncommon to find an aneurysm located at the origin of the AChA. In one of the adult specimens we did find such an aneurysm in a non-ruptured state. It is worthy to note that vascular aberrations frequently occur in the bifurcation site between CoA and M1 portion. Approximately $30-35 \%$ of the intracranial aneurysms were located in ACoA (Osborn, 1994). Needless to emphasize, endovascular embolization occupies a very important position in the treatment of aneurysms (Proust et al., 2009). The therapeutic microcatheter must be negotiated through the ICA and across the A1 portion of the anterior cerebral artery to reach the disease site in the ACoA. If A 1 portion of the anterior cerebral artery on either side is poorly developed, then the catheterization can be performed on the other side of A1 portion. MCA is the site of predilection not only for aneurysms, but also for arteriovenous malformations (AVM) and thrombus obstructions (Fernandez-Bouzas et al., 2006; Manawadu et al., 2008; Rinne et al., 1996). To be able to negotiate across this angle is the only recourse we have for either embolization transcatheter intraarterial thrombolytic therapy of the respective cerebral vascular disease(s).

Significance regarding vascular catheterization. Vascular intervention is the one technique which can give us both the diagnostic as well as the therapeutic aids by selectively planting a guidewire or a catheter in the target blood vessel through the vascular pathways. How precisely and rapidly we can introduce the therapeutic catheter into the target blood vessel(s) is what determines the possible worthy as well as unworthy outcomes; thus making this act the most crucial part of the required intervention. However, studies concerning catheterization techniques, which are well backed by concurrent researches in the cerebral vascular anatomy, have been very few. Particularly, studies pertaining to the vascular bending, bifurcation angle formation and the direction of the angles have not been reported. To our knowledge, this is the first study in which the bifurcation angle formation, the direction of the angles, bending and variation for all the levels of blood vessels that we come across during any possible cerebral vascular intervention have been systematically investigated and reported. The results 
obtained in this study provide morphological guidelines regarding diagnostic and therapeutic approaches, which can be applied by both the studying and practicing interventionists. These results are also instrumental to increase the speed and precision of catheterization, reduce associated radiation hazard, decrease iatrogenic damage to the endothelial membrane in the bifurcation angles, and avoid the occurrence of severe complications.

FAN, F.; XIANG, T.; TSAUO, T.; XIE, X. \& LI, X. Comparación del ángulo de arteria cerebral en adultos y fetos. Int. J. Morphol., 33(3):942-947, 2015.

RESUMEN: En este estudio generamos muestras de los vasos sanguíneos cerebrales humanos en veinticinco fetos y treinta y seis adultos. Fueron medidos y comprendidos los ángulos de aquellos vasos sanguíneos implicados frecuentemente en intervenciones de cateterismo.

PALABRAS CLAVE: Ángulo de la arteria cerebral; Cateterismo intervencionista; Muestras.

\section{REFERENCES}

Fernández-Bouzas, A.; Harmony, T.; Santiago-Rodríguez, E.; Ricardo-Garcell, J.; Fernández, T. \& Avila-Acosta, D. Schizencephaly with occlusion or absence of middle cerebral artery. Neuroradiology, 48(3):171-5, 2006.

Manawadu, D.; Quateen, A. \& Findlay, J. M. Hemicraniectomy for massive middle cerebral artery infarction: a review. Can. J. Neurol. Sci., 35(5):544-50, 2008.

Moore, K. L. The Developing Human: Clinically Oriented Embryology. 2nd ed. Philadelphia, W. B. Saunders Co., 1977. pp.322-7.

Mordasini, P.; Schroth, G.; Guzman, R.; Barth, A.; Seiler, R. W. \& Remonda, L. Endovascular treatment of posterior circulation cerebral aneurysms by using Guglielmi detachable coils: a 10year single-center experience with special regard to technical development. AJNR Am. J. Neuroradiol., 26(7):1732-8, 2005.

Osborn, A. G. Intracranial Aneurysms. In: Osborn, A. G. (Ed.). Diagnostic Neuroradiology. St. Louis, Mosby, 1994. pp.24883.

Proust, F.; Martinaud, O.; Gérardin, E.; Derrey, S.; Levèque, S.; Bioux, S.; Tollard, E.; Clavier, E.; Langlois, O.; Godefroy, O.; Hannequin, D. \& Fréger, P. Quality of life and brain damage after microsurgical clip occlusion or endovascular coil embolization for ruptured anterior communicating artery aneurysms: neuropsychological assessment. J. Neurosurg., 110(1):19-29, 2009.

Rinne, J.; Hernesniemi, J.; Niskanen, M. \& Vapalahti, M. Analysis of 561 patients with 690 middle cerebral artery aneurysms: anatomic and clinical features as correlated to management outcome. Neurosurgery, 38(1):2-11, 1996.

Turk, A. S.; Niemann, D. B.; Ahmed, A. \& Aagaard-Kienitz, B. Use of self-expanding stents in distal small cerebral vessels. AJNR Am. J. Neuroradiol., 28(3):533-6, 2007.
Wang, Y. Y.; Rosenfeld, J. V.; Lyon, S. M. \& O'Brien, B. J. Rapid development of a de novo intracranial aneurysm following carotid occlusion. J. Clin. Neurosci., 15(3):324-30, 2008.

\author{
Correspondence to: \\ Xiao Li \\ Department of Interventional Radiology \\ West China Hospital \\ Sichuan University \\ Chengdu, Sichuan Province \\ CHINA \\ Email: simonlixiao@gmail.com
}

Received: 17-10-2014

Accepted: 01-06-2015 